Infotech 2007 Abstract

An extremely low power quantum optical communication link for autonomous robotic explorers

Authors: John Lekki, Quang-Viet Nguyen, Tom Bizon, Binh Nguyen and Jun Kojima

One concept for planetary exploration involves using many small robotic landers that can cover more ground than a single conventional lander. In addressing this vision, NASA has been challenged in the National Nanotechnology Initiative to research the development of miniature robots built from nano-sized components. These robots have very significant challenges, such as mobility and communication, given the small size and limited power generation capability. The research presented here has been focused on developing a communications system that has the potential for providing ultra-low power communications for robots such as these. In this paper an optical communications technique that is based on transmitting recognizable sets of photons is presented. Previously pairs of photons that have an entangled quantum state have been shown to be recognizable in ambient light. The main drawback to utilizing entangled photons is that they can only be generated through a very energy inefficient nonlinear process. In this paper a new technique that generates sets of photons from pulsed sources is described and an experimental system demonstrating this technique is presented. This technique of generating photon sets from pulsed sources has the distinct advantage in that it is much more flexible and energy efficient, and is well suited to take advantage of the very high energy efficiencies that are possible when using nano scale sources. For these reasons the communication system presented in this paper is well suited for use in very small, low power landers and rovers. 


\title{
An extremely low power quantum optical communication link for autonomous robotic explorers
}

\author{
John Lekki ${ }^{1}$, Quang-Viet Nguyen ${ }^{2}$, Tom Bizon ${ }^{3}$, Binh Nguyen ${ }^{4}$ \\ NASA Glenn Research Center, Brook Park, Ohio, 44135 \\ and \\ Jun Kojima $^{5}$ \\ Ohio Aerospace Institute, Brook Park, Ohio, 44142
}

\begin{abstract}
[Abstract] In this paper a very low power optical communications system for miniature robots, as small as $1 \mathrm{~cm}^{3}$, is addressed. The communication system is a variant of photon counting communications. Instead of counting individual photons the system only counts the arrival of time coincident sets of photons. Using sets of photons significantly decreases the bit error rate because they are highly identifiable in the presence of ambient light. An experiment demonstrating reliable communication over a distance of 70 meters using less than a billionth of a watt of radiated power is presented. The components used in this system were chosen so that they could in the future be integrated into a cubic centimeter device.
\end{abstract}

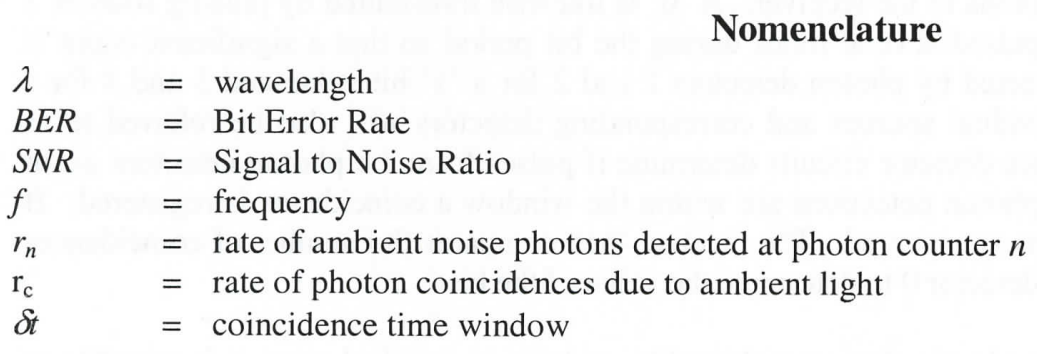

\section{Introduction}

$\mathrm{O}$ ne concept for planetary exploration involves using many small robotic landers that can cover more ground than a single conventional lander. In addressing this vision, NASA has been challenged in the National Nanotechnology Initiative to research the development of miniature robots built from nano-sized components ${ }^{1}$. These robots have very significant challenges, such as mobility and communication, given the small size and limited power generation capability. The research presented here has been focused on developing a communications system that has the potential for providing ultra-low power communications for robots such as these. In this paper an optical communications technique that is based on transmitting recognizable sets of photons is presented. In previous research, pairs of photons that have an entangled quantum state have been shown to be recognizable in ambient light even when the ambient background noise is significantly larger than the signal ${ }^{2,3,4,5}$. The main drawback to utilizing entangled photons is that they can only be generated through a very energy inefficient nonlinear process. In this paper a new technique that generates sets of photons from pulsed sources is described and an experimental system demonstrating this technique is presented. This technique of generating photon sets from pulsed sources has the distinct advantage in that it is much more flexible and energy efficient, and is well suited to take advantage of the

\footnotetext{
${ }^{1}$ Electronics Engineer, Optical Instrumentation and NDE branch, MS 77-1, AIAA Member.

${ }^{2}$ Research Aerospace Engineer, Combustion branch, MS 5-10.

${ }^{3}$ Electronics Engineer, Digital Communications branch, MS 54-8.

${ }_{5}^{4}$ Electronics Engineer, Digital Communications branch, MS 54-8.

${ }^{5}$ Senior Scientist, Combustion branch, MS OAI, AIAA Member.
} 
very high energy efficiencies that are possible when using nano scale sources. For these reasons the communication system presented in this paper is well suited for use in very small, low power landers and rovers.

\section{Communication system based on photon pairs}

The idea of using quantum optics for communication in very low signal-to-noise ratio (SNR) environments has been around for some time. Mandel ${ }^{2}$ first proposed one such system in 1984 and a demonstration experiment was performed by $\mathrm{Hong}^{3}$ in 1985. In this experiment, pairs of polarization entangled photons were generated and transmitted to the receiver over two beams of light that were on-off keyed with the data. The experiment demonstrated that highly correlated time coincident pairs of photons are highly recognizable in the presence of noise from ambient light. The general objective of these research activities is to develop a communication system based on time coincident photon pair communication that can eventually be built into a miniaturized transmitter that is very efficient in terms of the amount of electrical power used to transmit a bit of information. The nonlinear generation of quantum entangled photons is extremely energy inefficient and is not as feasible for usage in low power devices. In order for this communication method to be used it is necessary to develop a linear method for generating the signal photon pairs. There is no known method for linearly creating photon pairs that share a quantum state and are hence "Quantum Entangled" (QE). However, the QE communication method can be emulated. The QE communication method works because of the generation of inherently time coincident photon pairs that have highly correlated wavelengths and polarizations. It is possible to build a transmitter that linearly generates photons with these correlations. The primary subject of this research is a transmitter concept that can generate correlated photon sets, which look like entangled photons to a receiver, in a linear manner.

A time coincident photon communication system that linearly generates pairs of photons is shown as a block diagram in Figure 1. In this figure the details of how the beams are combined at the transmitter and likewise separated at the receiver are left out so that the basics of the signaling method can be understood. To transmit a ' 1 ' bit value, photon sources 1 and 2, which are separately identifiable by either wavelength, polarization or both, are pulsed at the same time to send pairs of photons to the receiver. A ' 0 ' is likewise transmitted by pulsing sources 3 and 4 at the same time. The sources are pulsed several times during the bit period so that a significant count is recorded at the receiver. The pairs are detected by photon detectors 1 and 2 for a ' 1 ' bit value and 3 and 4 for a ' 0 'bit value. Note that each of these individual sources and corresponding detectors will also be referred to as channels later in this paper. The coincidence detector circuits determine if pulses from the photon detectors occur within a coincidence time window. If the photon detections are within the window a coincidence is registered. If not within the window the photon detections are ignored. The receiver then compares the number of coincidences from coincidence detector 1 to coincidence detector 0 to determine the value of the bit.

In order to convey an idea of the power levels that are achievable, at least in an ideal case, it is possible to estimate the power per bit of information transmitted for an integrated device that utilizes this signaling method. This calculation is best done by starting with the required signal at the receiver. We know from the experiments performed up until now that the communication system has a reasonably low bit error rate if the number of coincidences registered by the receiver is greater than 10 per bit ${ }^{5}$. Assuming a detector has a quantum efficiency of $70 \%$ means that the receiver must collect at least 14 photon pairs per bit to register 10 per bit. For the transmitter, it can be assumed, somewhat hopefully, that a very advanced nanolaser will have a $50 \%$ conversion efficiency of electrical to optical energy ${ }^{6,7,8,9}$. The transmission loss is already known as the range is bounded to the distance at which the transmitted beam falls off to $50 \%{ }^{4}$. From these assumptions it can be estimated that 56 photon pairs / bit must be emitted from the transmitter to get 10 coincidences at the receiver. The electrical energy to generate these photons is equivalent to the energy of 112 photons per bit. The energy per bit is approximated by multiplying the number of photons, 112 , by the energy per photon equal to $2 \times 10^{-19} \mathrm{~J} /$ photon at 1 micron wavelength. This comes to $22 \times 10^{-18}$ joules. At a frequency of $100 \mathrm{kHz}$ the power this transmitter uses is equal to 2.2 pico watts (pW). 

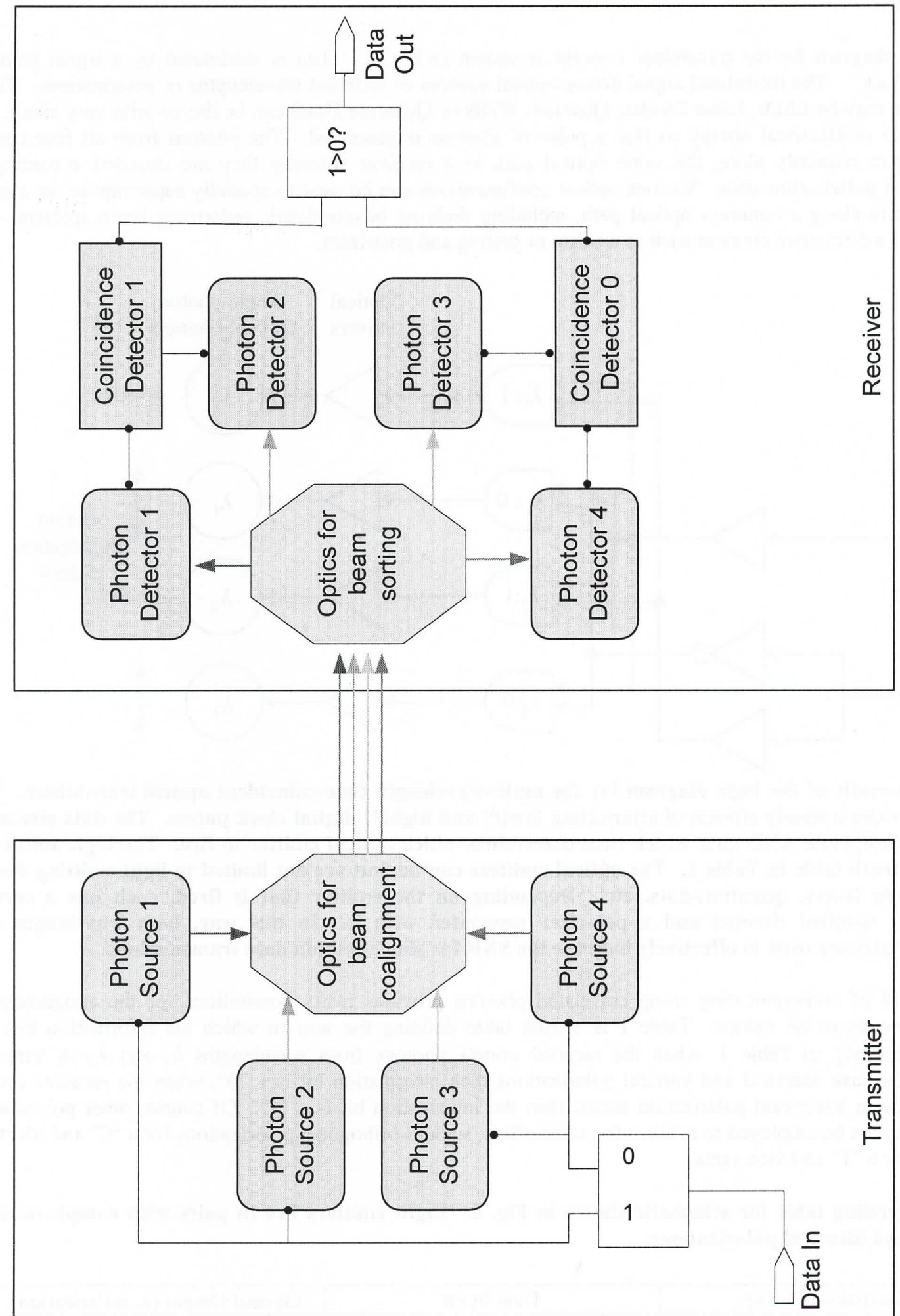

Figure 1. A time coincident photon communication system made from classical sources. 


\section{A. Transmitter}

The block diagram for the transmitter concept is shown in Fig. 2. Data is modulated by a signal from an oscillator or clock. The modulated signal drives optical sources of different wavelengths or polarizations. These sources (which may be LEDs, Laser Diodes, Quantum Wells or Quantum Dots) can be driven with very small, and very fast pulses of electrical energy so that a pulse of photons is generated. The photons from all four optical emitters are sent coaxially along the same optical path to a receiver whereby they are decoded according to wavelength and polarization state. Various optical configurations can be used to spatially superimpose the signals from all emitters along a common optical path, including dichroic beamsplitters, polarizing beam splitters, or a combination of a diffractive element such as a prism or grating and polarizers.

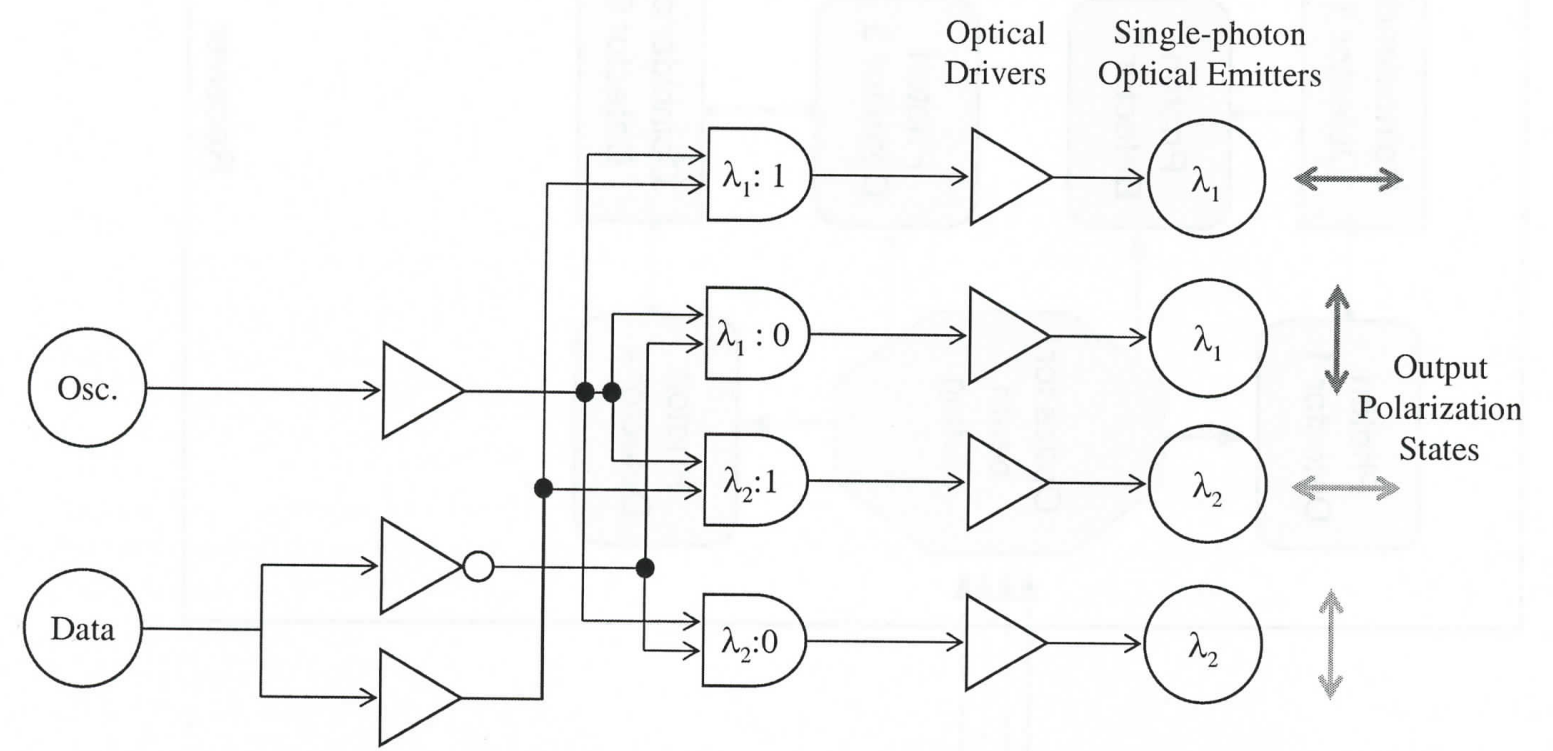

Figure 2. Schematic of the logic diagram for the multi-wavelength time-coincident optical transmitter. The oscillator provides a steady stream of alternating $\operatorname{low}(0)$ and high(1) digital clock pulses. The data stream is sent to the appropriate AND gate which then determines which optical emitter to fire. The logic states are shown in the truth table in Table 1. The optical emitters can be, but are not limited to light emitting diodes (LED) or diode lasers, quantum-dots, etc. Depending on the emitter that is fired, each has a certain wavelength or spectral channel and a polarizer associated with it. In this way, both wavelength and polarization states are used to effectively increase the SNR for single-photon data transmissions.

This method of communicating using correlated photons provides many possibilities for the assignment of physical parameters to bit values. Table 1 is a truth table defining the way in which the information bits are encoded. According to Table 1, when the receiver counts photons from wavelengths $\lambda_{1}$ and $\lambda_{2}$ in temporal coincidence that have identical and vertical polarizations then information bit is a "0"; when the receiver counts photons that are in horizontal polarization states, then the information bit is a "1". Of course, other polarization coding schemes can be employed to achieve the same effect, such as orthogonal polarizations for a "0" and identical polarizations for a " 1 " and vice versa.

Table 1. Bit coding table for schematic shown in Fig. 2. Light emitters fire in pairs with complementary wavelengths and identical polarizations.

\begin{tabular}{|c|c|c|}
\hline Oscillator (Clock) & Data Input & Optical Output $(\lambda$, polarization) \\
\hline 0 & any & 0 \\
\hline 1 & 0 & $\lambda_{1}$ Vertical, $\lambda_{2}$ Vertical \\
\hline 1 & 1 & $\lambda_{1}$ Horizontal, $\lambda_{2}$ Horizontal \\
\hline
\end{tabular}


Note that by use of additional logic gates, a system can be devised to send up to 2 bits $\left(2^{2}\right)$ of data to yield a possible of 4 combinations versus the 2 possible combinations shown in Table 1. The truth table for this configuration is shown in Table 2. For the communication schemes shown in Table 1 and Table 2, only two photons, one at each wavelength, have to be captured by the reciever. Since only two photons have to make it to the reciever, this implies that very few photons must be transmitted from the transmitter. The actual number of photons transmitted depends on the reciever size and quantum efficiency, the distance of the transmission and the directional capability of the transmitter.

Table 2. Truth table or logic chart for an alternate embodiment (not shown) but based on Fig. 2 where up to 2-bits ( 4 states) of information can be sent at a time through the use of perpendicular polarization states of $\lambda_{1}$ and $\lambda_{2}$.

\begin{tabular}{|c|c|c|}
\hline Oscillator (Clock) & Data Input & Optical Output $(\lambda$, polarization) \\
\hline 0 & any & 0 \\
\hline 1 & 0,0 & $\lambda_{1}$ Vertical, $\lambda_{2}$ Horizontal \\
\hline 1 & 1,0 & $\lambda_{1}$ Horizontal, $\lambda_{2}$ Vertical \\
\hline 1 & 0,1 & $\lambda_{1}$ Vertical, $\lambda_{2}$ Vertical \\
\hline 1 & 1,1 & $\lambda_{1}$ Horizontal, $\lambda_{2}$ Horizontal \\
\hline
\end{tabular}

The experimental version of the 1-bit transmitter was built using commercial off the shelf components. The components used were chosen with an emphasis on their potential to eventually be incorporated into a very energy efficient monolithic device that is appropriate for a miniature robot. For example laser diodes have been used along with collimating optics limited to an aperture diameter of $1 \mathrm{~cm}$. There were 2 laser diode sources at $669 \mathrm{~nm}$ and 2 at $673 \mathrm{~nm}$. They were arranged so that one laser of each wavelength was polarized vertically and the other was polarized horizontally. The lasers were pulsed 10 times per bit and the pulse duration of the laser pulses was approximately $10 \mathrm{~ns}$. This pulse duration is the lower limit of the commercially available diodes.

\section{B. Receiver}

In Figure 3 the receiver diagram is shown with more detail of the optical components. The receiver has a 50.8 $\mathrm{mm}$ diameter telescope to collect the photons and also collimate the received beam for the rest of the optical system. Immediately after the collection optics is a bandpass optical filter, denoted $\lambda_{l}$, that passes a 2 nm bandwidth centered at $669 \mathrm{~nm}$. The filter reflects the $673 \mathrm{~nm}$ photons which are guided to another $2 \mathrm{~nm}$ bandpass filter, denoted $\lambda_{2}$, centered at $673 \mathrm{~nm}$. With this set of filters the photons of wavelength 1 go to one set of photon counters (Geiger mode avalanche photodiodes with $50 \mathrm{~ns}$ dead time) and the photons of wavelength 2 to a separate set of photon counters. After the filter, the photons of wavelength 1 are separated by a Polarization Beam Splitter (PBS) into horizontal and vertical polarizations and then detected by photon counters. The photons of wavelength 2 are likewise separated by polarization and detected. The coincidence electronics determine which photon counters fire at the same time and thereby decode the data in bits of ones and zeros. The coincidence electronics have been built using a Field Programmable Gate Array operating at $400 \mathrm{MHz}$ and the coincidence time window is $5 \mathrm{~ns}$. The coincidence time window is influenced by the laser pulse duration. It cannot be too small compared to the pulse duration or a photon from one source early in the pulse will not be time coincident with another photon from its paired source late in the pulse. The number of coincidences due to noise photons is directly proportional to the coincidence time window ${ }^{10}$ and is approximately equal to

$$
r_{c} \approx \prod_{n} r_{n} \delta t
$$

where $n$ is the number of channels used for coincidences. Additional noise reduction would have been possible if a coincidence time of $1 \mathrm{~ns}$ were used but this would have resulted in significant signal loss because the laser pulses were of 10 ns duration. 


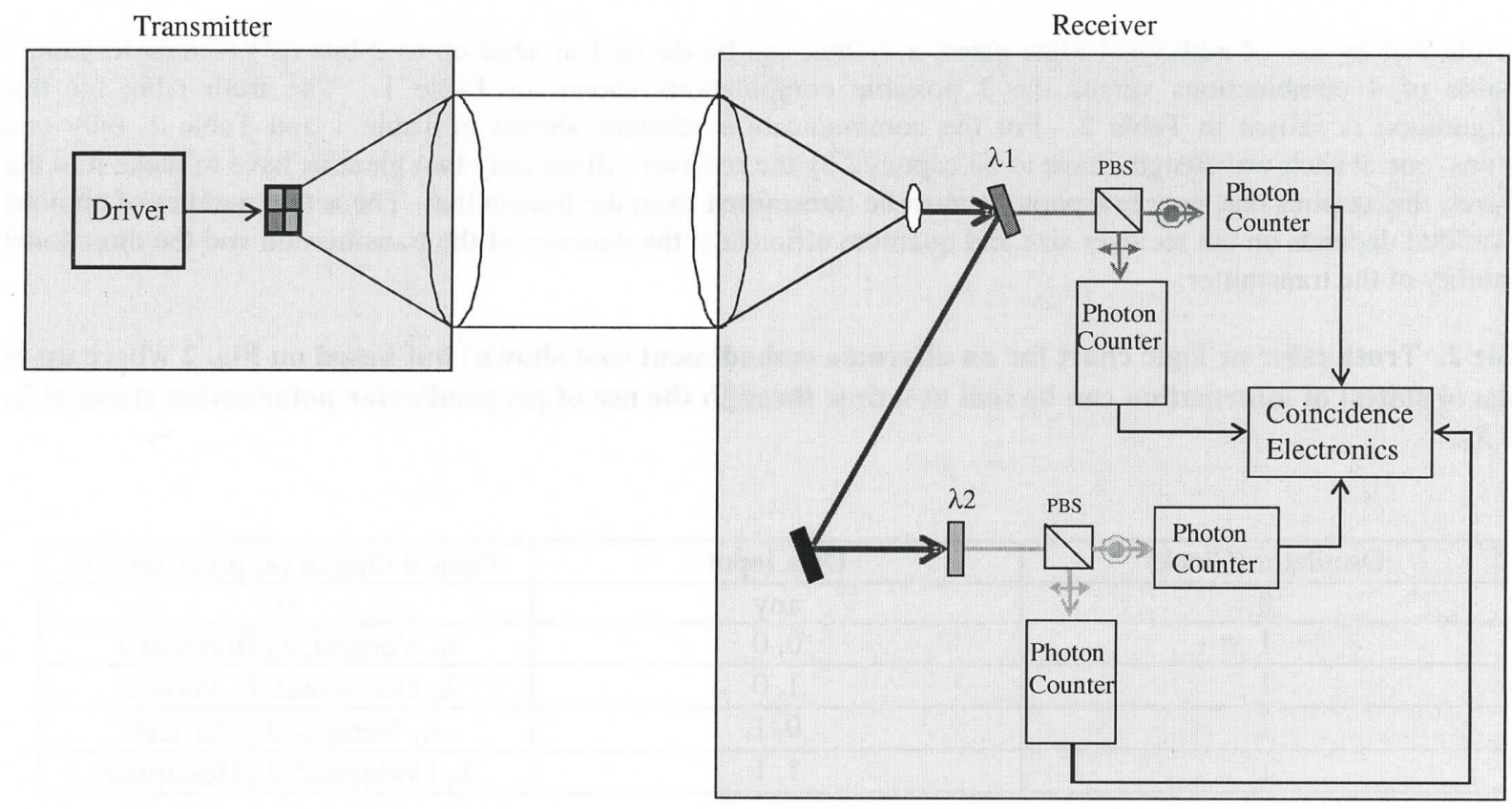

Figure 3. In this schematic the transmitter is shown working in conjunction with a receiver.

\section{Experimental test and results}

This system has been tested in indoor free space communication experiments. The experimental setup diagram is shown in Figure 4. The test was conducted in a hallway with the transmitter and receiver located next to one another. This allowed for the longest transmission distance and also made aligning the two easier. A white paper screen, with a small hole in it to allow for the transmitted beam to pass through, was placed in front of the transmitter. This screen provided a uniform reflector so that the background light seen by the receiver could be controlled by illuminating it with different brightness lights. In order to achieve the highest ambient light levels a fiber optic light source was located next to the beam path and directed towards the receiver. The highest background light levels were similar to outdoors on a sunny day while the lowest were similar to outdoors with heavy clouds. In this experiment the signal power for all configurations was attenuated by neutral density filters so that for each channel approximately 1 photon per pulse was detected at the receiver. The signal was kept constant for all experiments and the background light level was varied. Three experiment configurations were tested. The baseline configuration was just a single channel (no coincidence) that was used for traditional on/off keyed photon counting communication. In the second configuration photons were transmitted in time coincidence over 2 channels. A clock signal was transmitted and the bit values were encoded by on/off keying. The third configuration used 4 channels. In this configuration the data was keyed as shown in Table 1. The transmitted power for the single channel case was $180 \mathrm{pW}$, the 2 channel case was $360 \mathrm{pW}$ and for the 4 channel case $760 \mathrm{pW}$.

The conditions of this hallway were not ideal. There was a very significant temperature change between the room where the transmitter and receiver were located and the hallway the mirror was in. There was also significant turbulence in the hallway from the heating and cooling system. This caused a significant amount of beam wander, on the order of 1.5 to $2 \mathrm{~cm}$ linear displacement, which was probably very similar to what would be encountered outdoors. It was observed that the multiple beams did appear to stay together as they moved which was expected since the beams were at nearly the same wavelength. There were no active measures taken in the transmitter or receiver to counteract this beam wander so it should be noted that this was a significant contributor to the Bit Error Rate (BER).

In Figure 5 a sample of the raw data from the single channel (top) and two channel (bottom) configurations is shown. The single channel data plots photon detections as function of time. The two channel data plots photon coincidence detections over time. The data rate was $50 \mathrm{kHz}$ so there were 10 microseconds of 'on' with photon pulses and 10 microseconds 'off' without pulses. In the single channel case it can be seen that there are 10 
microsecond time periods with more photon counts and periods in between with less but the contrast between these is not very strong. In the 2 channel case the 10 microseconds of coincidence counts are readily apparent as there is almost no noise between them. The data clearly shows the noise immunity obtained by using coincident photon communication. The data however also shows some periods where the 2 channel coincidence counts are low. For example, in the 2 channel data the period between 30 and 40 microseconds there are only 4 coincidence counts. With some low counts such as these it is apparent that signal loss due to beam wander was probably one of the most significant sources of error.

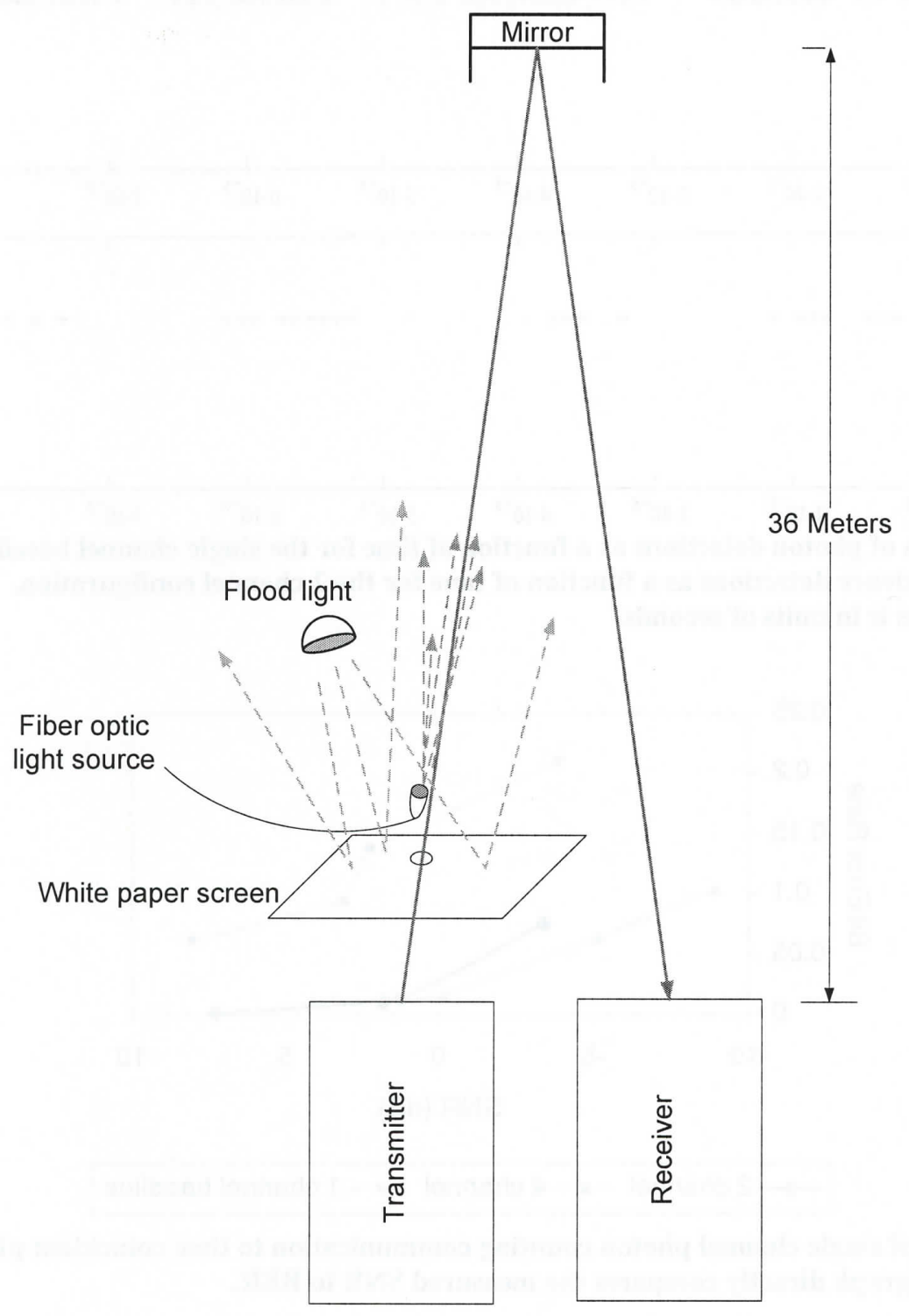

Figure 4. This figure shows the configuration of the ranged experiment. The experiment was performed in a hallway that allowed for a total of 72 meters of distance between the transmitter and receiver.

The data taken in these experiments has been tabulated and graphed in Figure 6 to show the BER as a function of SNR. The background light was varied to 4 different levels that were approximately equal for each configuration. The reason that the data points do not have the same SNR for each configuration is because the laser power levels 
could not be exactly matched and also by adding channels the amount of noise is increased linearly with the number of channels. A significant reduction of the BER is shown for the 2 channel coincidence compared to the single channel baseline. The 4 channel configuration also shows a significant reduction in BER from the baseline but the improvement from the 2 channel case was not as significant.
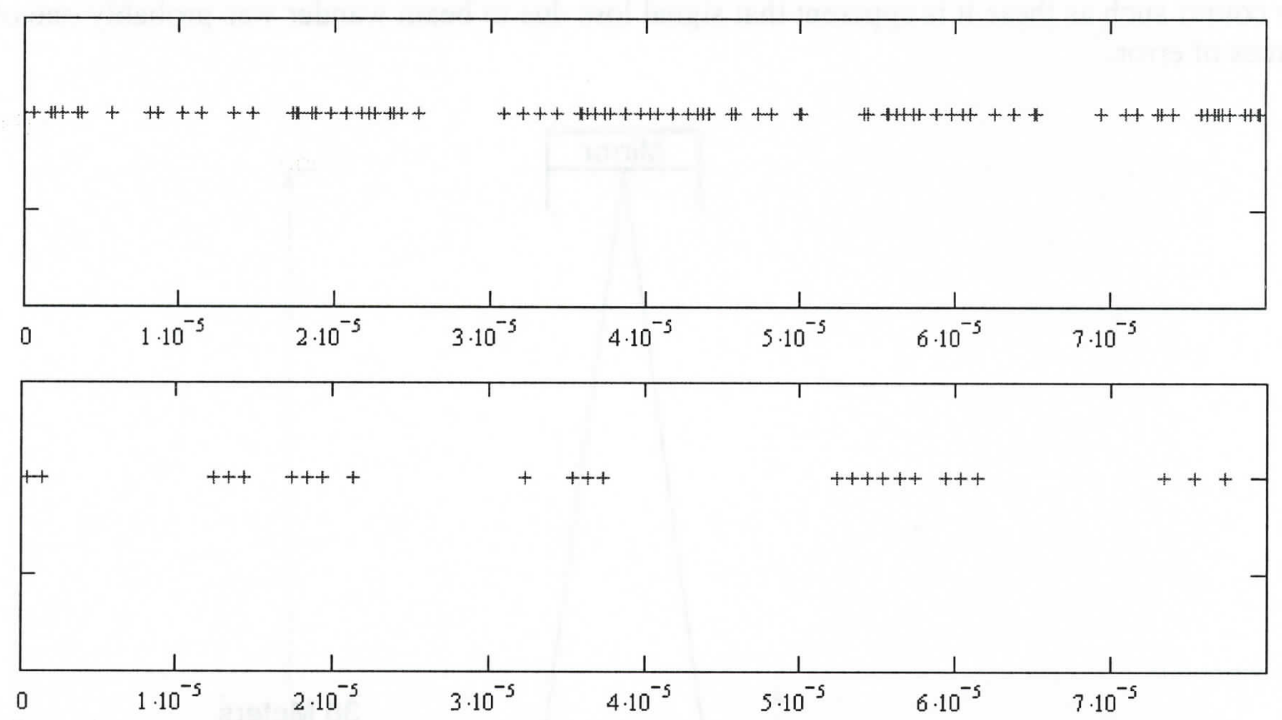

Figure5. Top: A graph of photon detections as a function of time for the single channel baseline. Bottom: A graph of photon coincidence detections as a function of time for the $\mathbf{2}$ channel configuration. The bottom axis for both of these graphs is in units of seconds.

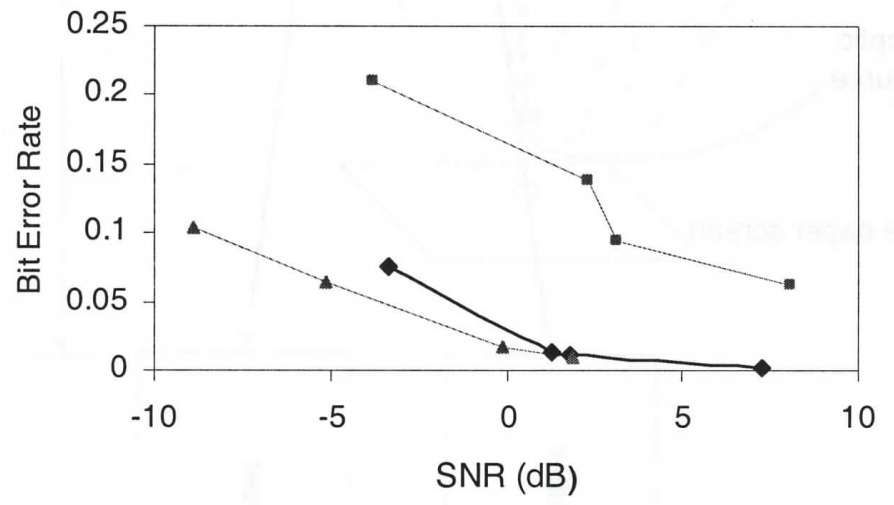

$\neg-2$ channel $\rightarrow 4$ channel $\ldots 1$ channel baseline

Figure 6. Comparison of single channel photon counting communication to time coincident photon counting communication. This graph directly compares the measured SNR to BER.

In order to more fairly evaluate this data a second graph has been generated in Figure 7 where the SNR is calculated in a way that compensates for the deflated SNR that results from inflated noise levels caused by additional channels. Here the signal is still the sum of the signal photons from each channel. The noise however is now calculated as the single channel average number of noise photons, rather than the sum of the noise photons from all channels. In Figure 7 we see that again a significant improvement comes from using the 2 channel photon coincidence as apposed to a single channel photon counting system. A 5 to $7 \mathrm{db}$ improvement has been measured in these tests. The data however does not show an improvement of the 4 channel system over the 2 channel system. In this case the 4 channel system would probably have to be coded with multiple bits, as in table 2 , for a given bit period to see an improvement. These results could be greatly improved by using transmitters that are capable of operating at a $1 \mathrm{~ns}$ pulse rate while operating the coincidence circuit at a matching $1 \mathrm{~ns}$ coincidence time. 


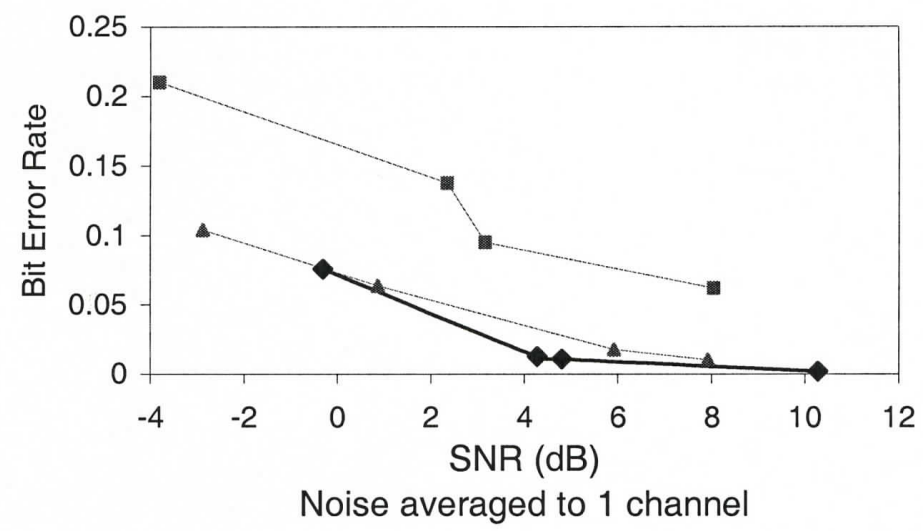

$\neg-2$ channel $\_4$ channel $\rightarrow 1$ channel baseline

Figure 7. In this graph the signal-to-noise ratio has been calculated with the signal summed for all of the channels used in a particular configuration. The noise however was calculated as an average for a single channel.

\section{Conclusion}

In this paper a new low power optical communication system has been presented. In contrast to traditional photon counting the system is made significantly immune to background noise by only counting photon coincidences. Experiments conducted with this system have shown that a signal of just $360 \mathrm{pW}$ can have an effective range of 70 meters even in the presence of ambient light levels similar to what can be expected outdoors. Previously this type of communication method has been accomplished using non linear systems to generate quantum entangled photons. The system has been developed because it can more readily utilize the smallest high efficiency sources that tend to have low total power levels. With sources such as these, the transmitter electrical energy can be as low as a billionth of a watt, which would overcome a significant hurdle for miniature robotic explorers.

\section{References}

${ }^{1}$ National Science and Technology Council, Committee on Technology, Subcommittee on Nanoscale Science, Engineering and Technology, "National Nanotechnology Initiative: The Initiative and Its Implementation Plan", July 2000, Washington D.C.

${ }^{2}$ Mandel, L., "Proposal for almost noise-free optical communication under conditions of high background", J. Opt. Soc. Am. B, Vol 1, March 1984, p. 108-110.

${ }^{3}$ Hong, C., Friberg, S. and Mandel, L., "Optical communication channel based on coincident photon pairs", Applied Optics 24(22), 3877-3882 (1985).

${ }^{4}$ Jackson, D., Hockney, G. and Dowling, J., "Quantum communication noise rejection using correlated photons", Journal of Modern Optics 49, 2383-2388 (2002).

${ }^{5}$ Lekki, J. D., Nguyen, Q., Nguyen, B. and Hizlan, M., "Quantum Optical Communication for Micro Robotic Explorers", Proceedings of the AIAA Infotech@Aerospace Conference, Arlington, Va., September (2005).

${ }^{6}$ Gourley, P., "Nanolasers", Scientific American Magazine; March 1998, p. 56-61.

${ }^{7}$ Miyazawa, T., Tatebayashi, J., Nakaoka, T., Ishida, S., Iwamoto, S., Takatsu, M., Arakawa, Y., Hirose, S., Takemoto, K., Usuki, T. and Yokoyama, N., "Electroluminescence from a Single Quantum Dot at Telecommunication Wavelength", Proceedings of CLEO / QELS conference, Baltimore (2005).

${ }^{8}$ Arakawa, Y., "Connecting the Dots", OEMagazine, January, 18-20 (2002).

${ }^{9}$ Mao, S. S., "Nanolasers: Lasing from Nanoscale Quantum Wires", Int. J. of Nanotechnology 1(1/2), $42-85$ (2004).

${ }^{10}$ Mandel, L. and Wolf, E., "Coherence Properties of Optical Fields,” Rev. Mod. Phys. 37, 231 (1965). 